\section{Adult-induced genetic ablation distinguishes PDGFB roles in blood-brain barrier maintenance and development}

\author{
Elisa Vazquez-Liebanas' ${ }^{D}$, Khayrun Nahar', \\ Giacomo Bertuzzi', Annika Keller $\left.{ }^{2}{ }^{(}\right)$, Christer Betsholtz ${ }^{1,3}$ and \\ Maarja Andaloussi Mäe' ${ }^{\mathbb{C}}$
}

Journal of Cerebral Blood Flow \& Metabolism 2022, Vol. 42(2) 264-279 (C) The Author(s) 2021 (c) (1)

Article reuse guidelines: sagepub.com/journals-permissions DOI: $10.1177 / 0271678 \times 211056395$ journals.sagepub.com/home/jcbfm (S)AGE

\begin{abstract}
Platelet-derived growth factor B (PDGFB) released from endothelial cells is indispensable for pericyte recruitment during angiogenesis in embryonic and postnatal organ growth. Constitutive genetic loss-of-function of PDGFB leads to pericyte hypoplasia and the formation of a sparse, dilated and venous-shifted brain microvasculature with dysfunctional blood-brain barrier (BBB) in mice, as well as the formation of microvascular calcification in both mice and humans. Endothelial PDGFB is also expressed in the adult quiescent microvasculature, but here its importance is unknown. We show that deletion of $P d g f b$ in endothelial cells in 2-months-old mice causes a slowly progressing pericyte loss leading, at $12-18$ months of age, to $\approx 50 \%$ decrease in endothelial:pericyte cell ratio, $\approx 60 \%$ decrease in pericyte longitudinal capillary coverage and $>70 \%$ decrease in pericyte marker expression. Similar to constitutive loss of Pdgfb, this correlates with increased BBB permeability. However, in contrast to the constitutive loss of Pdgfb, adult-induced loss does not lead to vessel dilation, impaired arterio-venous zonation or the formation of microvascular calcifications. We conclude that PDFGB expression in quiescent adult microvascular brain endothelium is critical for the maintenance of pericyte coverage and normal BBB function, but that microvessel dilation, rarefaction, arterio-venous skewing and calcification reflect developmental roles of PDGFB.
\end{abstract}

\title{
Keywords
}

Adult brain vasculature, blood-brain barrier, blood-retina barrier, PDGFB, pericyte, platelet-derived growth factor, vascular smooth muscle cell, permeability

Received I2 May 202I; Revised 28 September 202I; Accepted 29 September 2021

\section{Introduction}

Platelet-derived growth factor B (PDGFB), originally identified as a constituent of PDGF molecules in blood serum and human platelets, is one of four PDGF chains (PDGF A-D) that make up the PDGF family of homo- and heterodimeric ligands. ${ }^{1}$ The $P d g f b$ gene consists of seven exons. Exons 4 and 5 encode the growth factor core domain and thereby the major part of the mature protein. A carboxy-terminal motif mediating heparan sulfate proteoglycan (HSPG) binding of PDGFB is encoded by exon $6 .^{2,3}$ Biologically active PDGFB ligands are usually disulfide-linked PDGF-BB homodimers and exert their major biological effects via the tyrosine kinase receptor plateletderived growth factor receptor $\beta$ (PDGFRB).
The physiological roles of PDGFB have been studied mainly during development. Embryonic deletion of either of the $P d g f b$ or $P d g f r b$ genes in mice are

\footnotetext{
'Department of Immunology, Genetics, and Pathology, Rudbeck Laboratory, Uppsala University, Uppsala, Sweden

${ }^{2}$ Department of Neurosurgery, Clinical Neurocentre, Zürich University Hospital, Zürich University, Zürich, Switzerland

${ }^{3}$ Department of Medicine Huddinge, Karolinska Institute, Campus

Flemingsberg, Huddinge, Sweden
}

\section{Corresponding author:}

Maarja Andaloussi Mäe, Division of Vascular Biology, Rudbeck Laboratory, Department of Immunology, Genetics and Pathology, Uppsala University, 75I 85 Uppsala, Sweden.

Email: maarja.andaloussi_mae@igp.uu.se 
perinatally lethal due to widespread hemorrhage and general edema. ${ }^{4,5}$ The complete loss of PDGFB or PDGFRB proteins results in failure of mural cell (pericyte and vascular smooth muscle cell (VSMC)) proliferation and recruitment to the developing vasculature, which causes blood vessel dilation and dysfunction. ${ }^{6,7}$ Furthermore, loss-of-function mutations in the $P D G F B$ and $P D G F R B$ genes cause primary familial brain calcification (PFBC), a dominantly inherited age-dependent neurodegenerative disease, characterized by bilateral vessel-associated calcifications in the basal ganglia. ${ }^{8,9}$

The $P d g f b$ retention motif knockout mouse $\left(P d g f b^{\text {ret/ret }}\right)$ is a hypomorphic $P d g f b$ model that has been used to study postnatal effects of pericyte loss, particularly in the central nervous system (CNS) vasculature. $^{9-15}$ The $P d g f b^{\text {ret }}$ mutation renders a biologically active PDGFB protein, which however, due to deletion of the exon 6-encoding domain and resulting inability of the growth factor to bind extracellular HSPGs, fails to shape appropriate peri-endothelial PDGFB gradients that are required to stimulate proper recruitment of pericytes to the developing vasculature. ${ }^{16,17}$ As a consequence, fewer pericytes are recruited, which may also be less well integrated in the vessel wall. ${ }^{12} P d g f b^{\text {ret/ret }}$ mice are adult viable but show severely reduced brain pericyte coverage $(25 \%$ longitudinal coverage as compared with $P d g f b^{\text {ret/ }}+$ controls) and a substantially increased capillary diameter. ${ }^{10,15} P d g f b^{\text {ret/ret }}$ mice retain a general Wnt-induced blood-brain barrier (BBB)-specific gene expression profile but acquire a venous-shifted endothelial profile, upregulate expression of numerous growth factors and cytokines, and display angiogenic sprouting and impaired BBB function caused by increased transcytosis and focal hotspot leakage sites with disrupted endothelial junctions. ${ }^{10,15}$ Additionally, adult $P d g f b^{\text {ret } / \text { ret }}$ mice develop microvascular calcifications in deep brain regions similarly to PFBC patients. ${ }^{9,13,14}$ Importantly, endothelial expression of PDGFB protects mice from vessel calcification, ${ }^{9}$ indicating that PDGFB has homeostatic functions in the vessel wall in addition to pericyte recruitment.

PDGFB is expressed by several cell types in the brain. ${ }^{1,18,19}$ In order to analyze the vascular functions of PDGFB, previous studies have used constitutive Tiel-Cre to ablate $P d g f b$ during embryonic development. $^{20,21}$ These mice were viable and reached adulthood, but deletion of $P d g f b$ was partial and inter-individually variable, with $20-90 \%$ recombination efficiencies correlating with $30-90 \%$ pericyte loss in the $\mathrm{CNS}^{20}$. Likewise, early postnatal deletion of endothelial-specific PDGFB accomplished using tamoxifen-induced $C d h 5$-CreERT2 mediated knockout strategy (P5-P7), demonstrated a crucial role for PDGFB in recruiting pericytes to developing retinal capillaries. $^{22}$ Thus, both constitutive and developmentally-induced PDGFB knockouts have invariably displayed $\mathrm{BBB}$ and blood-retinal barrier defects that correlate in severity with the degree of pericyte hypoplasia, together demonstrating that PDGFB signaling through PDGFRB is critical for developmental assembly of functional blood vessels. However, if PDGFB also has a homeostatic role in fully developed CNS blood vessels is less understood. PDGFB continues to be expressed in adult brain endothelium. ${ }^{18}$ Retinal vasculature in mice with postnatallyinduced (at 8-9 weeks) endothelial $P d g f b$ knockout maintained pericyte coverage and displayed an intact blood-retinal barrier one month later, ${ }^{22}$ however, the long-term consequences were not addressed.

In this study, we investigated whether endothelial PDGFB is required for pericyte and VSMC survival and maintenance in the fully developed and quiescent adult brain and retinal vasculature over a prolonged time period. We deleted $P d g f b$ in 2-month-old mice and analyzed them at 4-, 12- and 18-months of age. Our results show that loss of endothelial PDGFB leads to a slowly progressing loss of mural cells eventually triggering BBB permeability to both low and high molecular weight tracers. However, we did not find certain other phenotypes associated with developmental pericyte loss, such as increased capillary diameter and microvascular calcification. Our data thereby distinguish between developmental and homeostatic roles for PDGFB in the brain vasculature.

\section{Material and methods}

\section{Experimental animals}

Endothelial-specific tamoxifen-inducible $P d g f b$ knockout mice (referred to as $P d g f b^{\mathrm{iECKO}}$ ) were obtained by crossing $P d g f b^{\text {flox/flox } 20}$ or $P d g f b^{\text {flox } /-4,20}$ mice with endothelial specific $C d h 5$ (PAC)-CreERT2 mice. ${ }^{23}$ For all experiments, Cre negative mice with either $P d g f b^{\text {flox/flox }}$ or $P d g f b^{\text {flox/+ }}$ genotypes and/or C57BL6 mice are referred to as controls (Ctrl). Gene deletion was induced by daily tamoxifen (cat. \#T5648, SigmaAldrich) administration $(100 \mu \mathrm{l}$ of a $20 \mathrm{mg} / \mathrm{ml}$ solution in $90 \%$ corn oil (cat. \#C 8267 , Sigma-Aldrich) and $10 \%$ ethanol) by oral gavage for 5 consecutive days at approximately 2 months of age. To minimize potential confounders, all animals within each experiment were treated with the same batch of Tamoxifen. Mice were sacrificed for brain analysis after 2 weeks ( 1 litter, Ctrl $\mathrm{n}=4, P d g f b^{\mathrm{iECKO}} \mathrm{n}=5$ ) and approximately 2 months (7 litters, Ctrl $\mathrm{n}=21, \quad P d g f b^{\text {iECKO }} \mathrm{n}=25$ ) after 
induction (referred to as "young" mice) or approximately 10 (5 litters, Ctrl $\left.\mathrm{n}=15, P d g f b^{\text {iECKO }} \mathrm{n}=14\right)$ and 16 months ( 1 litter, $\left.\operatorname{Ctrl} \mathrm{n}=1, P d g f b^{\mathrm{iECKO}} \mathrm{n}=4\right)$ after induction (referred to as "old" mice). Two of the control animals in 16 months after induction group died before analysis, leaving us with only one littermate control for the comparison. For retinal analysis mice were taken at approximately 2 months (1 litter, Ctrl $\left.\mathrm{n}=3, P d g f b^{\mathrm{iECKO}} \mathrm{n}=4\right)$ and 16 months (1 litter, Ctrl $\left.\mathrm{n}=1, P d g f b^{\text {iECKO }} \mathrm{n}=2\right)$ after induction. In addition, we analyzed non-induced mice ( 2 litters, Cre negative $\mathrm{n}=6$, Cre positive $\mathrm{n}=6$ ) to rule out the possibility of genetic background influencing the phenotype. For RNA in situ analysis 3-months-old C57BL/6 $(\mathrm{n}=3)$ mice were used.

Animal housing, as well as the experiments performed, were in accordance with Swedish legislation and were approved by the Uppsala Committee on the Ethics of Animal Experiments (permit numbers C224/ 12, C115/15, C111515/16). Experiments have been reported in compliance with the ARRIVE 2.0 guidelines. ${ }^{24}$ All efforts were made to minimize animal suffering. Both male and female mice were used in the experiments, and the studied phenotypes did not differ between sexes. All mice were back-crossed at least 10 generations to C57BL/6 background. No animals were excluded from the analyses. All quantifications were carried out with the investigator blinded to genotype.

\section{In situ RNA hybridization}

In situ RNA hybridization was performed using RNAscope technology (ACDBio) following the manufacturer's protocol with minor modifications as previously reported. ${ }^{18}$ The fluorescent signal was captured using a Leica TCS SP8 confocal microscope (Leica Microsystems). The following RNAscope probes from ACDBio were used: Pdgfb (\#424651), Pecam1 (\#316721) and Tubb3 (\#423391). All images presented are 2D maximum intensity projections of $\sim 4 \mu \mathrm{m}$ z-stacks. According to the ACDBio, each mRNA molecule hybridized to a probe appears as a separate small fluorescent dot.

\section{Microvasculature isolation and quantitative PCR}

After perfusion with Hank's Balanced Salt Solution (HBSS, \#14025092, Life Technologies), one hemisphere of the brain was used for microvasculature isolation and qPCR and the other hemisphere was used for additional experiments (BBB integrity assessment, immunofluorescence stainings). The chopped tissue was incubated with $0.5 \mathrm{mg} / \mathrm{ml}$ of collagenase type 2 (\#C6885, Sigma) in Dulbecco's Modified Eagle's
Medium (DMEM, \#31885-023, Life Technologies) for 10 minutes at $37^{\circ} \mathrm{C}$. The suspension was neutralized with DMEM with $20 \%$ fetal bovine serum (FBS, \#10500-064, Life Technologies) and filtered through a $70 \mu \mathrm{m}$ mesh (\#352350, BD Biosciences). After centrifugation, the pellet was resuspended in DMEM with $0.5 \mathrm{mg} / \mathrm{ml}$ heparin (\#H3393-100KU, Sigma-Aldrich) to prevent coagulation. The cell suspension was incubated with sheep anti-rat magnetic Dynabeads (\#11035, Life Technologies) bound to rat anti-mouse PECAM1 antibody (\#AF3628, BD Pharmingen) for 60 minutes at $4^{\circ} \mathrm{C}$. Afterwards, the microvascular fragments were collected with a DynaMag-2 magnet and washed with DMEM containing $0.5 \mathrm{mg} / \mathrm{ml}$ of heparin and lysed in RLT buffer. RNA was extracted with the RNeasy Plus micro kit (\#74034, Qiagen). cDNA was synthesized using iScript RT Supermix (\#170-8841, BioRad) and quantified using Taqman assays (Applied Biosystems). The probes used are summarized in Suppl. Table 1, as well as the number of litters and animals analyzed. The expression levels of all genes were normalized to endogenous gene expression levels of Gapdh.

\section{Immunofluorescence stainings}

Mice were perfused transcardially under full anesthesia (Hypnorm-Midazolam or Ketamine-Xylazine mixture) with HBSS, followed by $4 \%$ buffered formaldehyde (Formalin, \#2178, Histolab). Brains and retinas were removed and post-fixed in formalin for 4 and $2 \mathrm{~h}$, respectively, at $4^{\circ} \mathrm{C}$. Fifty to seventy-five $\mu \mathrm{m}$ brain sagittal vibratome sections and retinas were incubated in blocking/permeabilization solution ( $1 \%$ bovine serum albumin (BSA), 0.75\% TritonX-100 (\#T8787, SigmaAldrich)), 5\% normal donkey serum (\# 017-000-121, Jackson ImmunoResearch) in PBS) overnight at $4^{\circ} \mathrm{C}$, followed by incubation in primary antibody solution for $48-72 \mathrm{~h}$ at $4^{\circ} \mathrm{C}$, and subsequently in secondary antibody solution overnight at $4^{\circ} \mathrm{C}$. Sections were mounted in Prolong Gold anti-fade reagent (\#P36930, Thermo Fischer Scientific). A detailed list of the primary antibodies used for this study can be seen in Suppl. Table 2 . Secondary antibodies from donkey for multiple labelling conjugated with the appropriate fluorescent dyes were from ThermoScientific or Jackson ImmunoResearch. As a negative control the sections were incubated with secondary antibodies only. Cell nuclei were visualized with Hoechst 33342 (Hoecsht, \#H3570, ThermoFisher). Image processing was done using Fiji, Adobe Photoshop 2020 (21.2.3), and Adobe Illustrator 2020 (24.3). All immunofluorescence images were taken with Leica TCS SP8 and presented as maximum intensity projections of confocal z-stacks. 


\section{Quantification of pericyte coverage, endothelial cell number and length}

From cerebral cortex, two $60-75 \mu \mathrm{m}$-thick vibratome sections per mouse, five $10.17 \mu \mathrm{m}$-thick $\mathrm{z}$-stacks per section and four $6.78 \mu \mathrm{m}$ z-stacks for each vascular plexus in half a retina were obtained with a Leica TCS SP8 and 40x objective. The sections were coimmunolabeled with PECAM1, ANPEP, ERG and the nuclei were visualized with Hoechst. Pericyte coverage was quantified as previously reported. ${ }^{25}$ In the brain, all the ERG+ cells per field were counted as well as all the ANPEP+ cell bodies colocalizing with Hoechst. The ratio of ERG + cells over ANPEP + cell was considered as the EC/PC ratio per each field.

To study regional differences in pericyte coverage, three $10.17 \mu \mathrm{m}$-thick z-stacks from one section were obtained. The analysis was done in the hippocampus, thalamus, midbrain, cerebellar center and corpus callosum. Furthermore, for the pericyte longitudinal coverage analysis and vascular length from the midbrain, two litters of old mice $\left(P d g f b^{\text {iECKO }} \mathrm{n}=8, C \operatorname{trl} \mathrm{n}=3\right.$ ) were included. In this case, five $\mathrm{z}$-stacks per section from two sections were used for the analysis.

\section{Analysis of apoptosis}

At 2 weeks after induction, two $30 \mu \mathrm{m}$-thick sagittal vibratome sections per mouse brain were collected and stained for cleaved-CASPASE3 in combination with ANPEP and PECAM1. Hoechst was used to localize the nuclei. All cleaved-CASPASE3 positive cells present on each section were captured and counted with a Leica TCS SP8 and 63x objective. Co-immunolabeling of PECAM1 and ANPEP together with cleaved-CASPASE3 positive cells allowed the localization of apoptotic endothelial cells and pericytes or VSMC, respectively. All cleaved-CASPASE3 positive cells that did not co-localize with either PECAM1 or ANPEP were considered as "other cell type" of apoptotic cells. Representative images are presented as maximum intensity projections of confocal z-stacks.

\section{Blood-brain barrier and retina integrity assessment}

Blood-brain barrier integrity analyses with Alexa Fluor 555 cadaverine (\#A30677, Life Technologies, $11 \mu \mathrm{g} / \mathrm{g}$ body weight) were carried out as previously. ${ }^{15}$ Results were expressed as fold change of the controls.

For blood-retina barrier integrity assessment, Alexa Fluor 555 cadaverine injected mice were perfused $2 \mathrm{~h}$ after tracer administration first with HBSS then followed by $4 \%$ buffered formaldehyde. The retinas were dissected and post-fixed in $4 \%$ buffered formaldehyde for $2 \mathrm{~h}$, at $4^{\circ} \mathrm{C}$ and analyzed with Leica TCS SP8.

\section{TMR-Dextran hotspot leakage quantification}

$70 \mathrm{kDa}$ Dextran conjugated to tetramethylrhodamine $(125 \mu \mathrm{g} / \mathrm{g}$ body weight) (TMR, \#D1818, ThermoFisher) was injected intravenously into the tail vein of tamoxifen-induced 12-month-old mice $16 \mathrm{~h}$ before sacrifice. $60-65 \mu \mathrm{m}$-thick vibratome brain sections were co-immunostained for PECAM1, CLDN5 and CDH5. Two sections per mouse were analyzed and all the present $70 \mathrm{kDa}$ TMR-Dextran hotspots were imaged and counted with a Leica TCS SP8 and 63x objective. Representative images are presented as maximum intensity projections of confocal z-stacks.

\section{Statistical analysis}

GraphPad Prism v5.01 was used for all the statistical analysis. Data is presented as geometrical mean \pm geometrical SD. When zero values are present, the data is presented as mean $\pm \mathrm{SD}$. Shapiro-Wilk and Kolmogorov-Smirnov test were used to study normality, when there were no zero values or $\mathrm{n}$ was greater than 1 . For normally distributed data with two group comparisons two-tailed, unpaired student's $t$ test was performed. For unevenly distributed data, the nonparametric Mann Whitney U test was used. For multiple comparisons with normally distributed data, Tukey's multiple comparison test was used, whereas for unevenly distributed data, the nonparametric Kruskal-Wallis multiple comparison test was used. For all tests, $P \leq 0.05$ was considered statistically significant. For group size determination, no formal prior power calculations were performed. The practical circumstances determined the number of mice in each experiment. For all the images included across the manuscript, the most representative examples reflecting the typical phenotype were selected.

\section{Results}

\section{Pdgfb gene deletion efficiency in adult $P d g f b^{i E C K O}$ mice}

Previous work has established that $P d g f b$ is expressed by endothelial cells in the developing vasculature, ${ }^{7}$ in particular in tip cells of angiogenic sprouts and in developing arterial endothelial cells. ${ }^{6,26}$ More recent single-cell RNA sequencing data show that endothelial $P d g f b$ expression is maintained in the adult mouse brain, and that endothelial cells are the major site of $P d g f b$ expression in this organ. However, $P d g f b$ expression was also found to a lower extent in VSMC, microglia and certain type of neurons. ${ }^{18,19}$ Single-cell RNA sequencing data further suggests that endothelial $P d g f b$ 
expression occurs at similar levels in arteries, capillaries and veins. ${ }^{18}$ Due to a lack of reliable anti-PDGFB antibodies we performed RNA in situ hybridization experiments on adult mouse brains, which confirmed co-expression of $P d g f b$ with the endothelial cell marker Pecam 1 mRNA in vascular structures (Suppl. Fig. 1A), as well as $P d g f b$ and the neuronal marker Tubb3 in certain neurons (Suppl. Fig. 1B).

In order to gain insights into the role of endothelium-derived PDGFB in the adult quiescent brain vasculature, we created endothelial-specific $P d g f b$ knock-out mice using a tamoxifen-inducible strategy by crossing either $P d g f b^{\text {flox/flox,20 }} P d g f f^{f l o x /+}$ or $P d g f f^{\text {flox } /-} 7$ mice with $C d h 5$-CreERT2 mice. $^{23}$ This breeding strategy gave rise to litters including Cre-positive $P d g f b^{\text {flox/flox }}$ or $P d g f b^{\text {flox/- }}$ mice, which were collectively used as conditional endothelial cell PDGFB knock-outs $\left(P d g f b^{\text {iECKO }}\right)$, as well as Crenegative $P d g f b^{\text {flox/flox }}$ or $P d g f b^{f l o x /+}$ pups, which were collectively used as littermate controls (Ctrl). $P d g f b^{\text {iECKO }}$ and control mice were administered tamoxifen once per day for five consecutive days at 2 -months of age and sacrificed at 4-months of age (referred to as young age), or 12- or 18-months of age (collectively referred to as old age) (Figure 1(a)). Quantitative PCR analysis of microvascular fragments revealed a substantial reduction in $P d g f b$ transcript levels in both young (8\% remaining, Figure $1(\mathrm{~b}))$ and old $P d g f b^{\mathrm{iECKO}}$ mice (4\% remaining, Figure $1(\mathrm{~b})$ ) compared with controls.

Analysis of 2-months-old Cre-positive and Crenegative mice without tamoxifen induction showed no difference in pericyte longitudinal coverage (Suppl. Fig. 1C, D), endothelial cell:pericyte ratio (Suppl. Fig. 1E), vascular length (Suppl. Fig. 1F) or total number of ERG+ endothelial cells per field (Suppl. Fig. 1G). Additionally, we did not detect any active angiogenesis by staining for tip cell marker MCAM ${ }^{15}$ together with PECAM1, which indicates that the brain vasculature is largely quiescent at 2 months of age (Suppl. Fig. 1H).

\section{Endothelium-derived PDGFB is necessary for maintenance of pericyte coverage in adult mouse brain vasculature}

Reduced density of mural cells is a well-characterized consequence of $P d g f b$ deletion in the developing vasculature. $6,7,10,12,15,22$ To assess if inducible ablation of endothelium-derived $P d g f b$ has an effect on brain mural cell coverage after the brain vasculature is fully developed in adults, we investigated capillary pericyte coverage in control and $P d g f b^{\text {iECKO }}$ mice at both young and old age. Low magnification images revealed a substantial loss of ANPEP-positive pericytes in
$P d g f b^{\text {iECKO }}$ mice compared with controls already at 4 months of age (2 months after $P d g f b$ deletion), and this became more pronounced in 12- and 18-months-old mice (Figure 1(c)). At high magnification, the pericyte processes in $P d g f b^{\mathrm{iECKO}}$ mice appeared thinner, partly detached and with discontinuous ANPEP staining (Figure 1(d), white arrowheads). Pericytes in $P d g f b^{\mathrm{iECKO}}$ mice extended shorter processes compared with control mice, leaving stretches of the vasculature uncovered (Figure 1(d), yellow arrowheads). In addition, we observed pericytes with altered cell body morphology and shorter foot-like cytoplasmic processes, which were not seen in control animals (Figure 1(d), asterisk).

Quantitative PCR analysis of isolated microvascular fragments showed significant reduction of several known pericyte markers, including $P d g f r b \quad(34 \%$ remaining), Anpep (38\%), Abcc9 (31\%) and Kcnj8 $(37 \%)$ in $P d g f b^{\text {iECKO }}$ when compared with littermate controls in 4-months-old mice (Figure 1(e)) and these markers were also decreased in old $P d g f b^{\text {iECKO }}$ mice when compared with controls (Pdgfrb $(45 \%)$, Anpep (29\%), Abcc9 (27\%) and Kcnj8 (32\%)) (Figure 1(e)). Immunofluorescence staining using anti-desmin (DES) and anti-PDGFRB antibodies confirmed that the pericyte markers expression in $P d g f b^{\mathrm{iECKO}}$ were reduced overall (Figure 1(f)).

Quantification of longitudinal capillary coverage by ANPEP-positive pericytes showed a significant reduction in pericyte coverage in the cerebral cortex with 4-month-old $P d g f b^{\text {iECKO }}$ mice $(74 \%$ coverage remaining) which was further reduced in 12-and 18-month-old mice $(43 \%$ coverage remaining) when compared with controls (100\%) (Figure 1(g)). Quantification of endothelial cell (EC) $\left(\mathrm{ERG}^{+}\right.$, Hoechst $\left.^{+}\right)$to pericyte (PC) ratio $\left(\mathrm{ANPEP}^{+}, \mathrm{Hoechst}^{+}\right)$demonstrated increase from controls (which had 4 EC per 1 PC) in both 4month-old (7 EC per 1 PC) and 12- and 18-month-old $P d g f b^{\text {iECKO }}$ mice (8 EC per 1 PC) (Figure 1(h)). In the 18-month-old litter, two of the control animals died before analysis, leaving us with only one littermate control for the comparison. In order to appreciate if there is further pericyte reduction in the 18-month-old litter, we quantified pericytes separately in 12- and 18-monthold brains. Pericyte longitudinal coverage was slightly decreased in 18-month-old mice $(33 \%$ coverage remaining) compared to 12 -month-old $P d g f b^{\text {iECKO }}$ mice (44\% coverage remaining) (Suppl. Fig. 2A), however the EC/PC ratio (8 EC per $1 \mathrm{PC}$ ) remained the same at both ages (Suppl. Fig. 2B). Total vascular length (Suppl. Fig. 2C) and total ERG+ endothelial cell numbers per field remained unaltered at all studied ages (Suppl. Fig. 2D). Additionally, we did not observe any obvious vessel dilation in $P d g f b^{\text {iECKO }}$ brains (Figure 1(c), (d) and (f)), an otherwise characteristic 


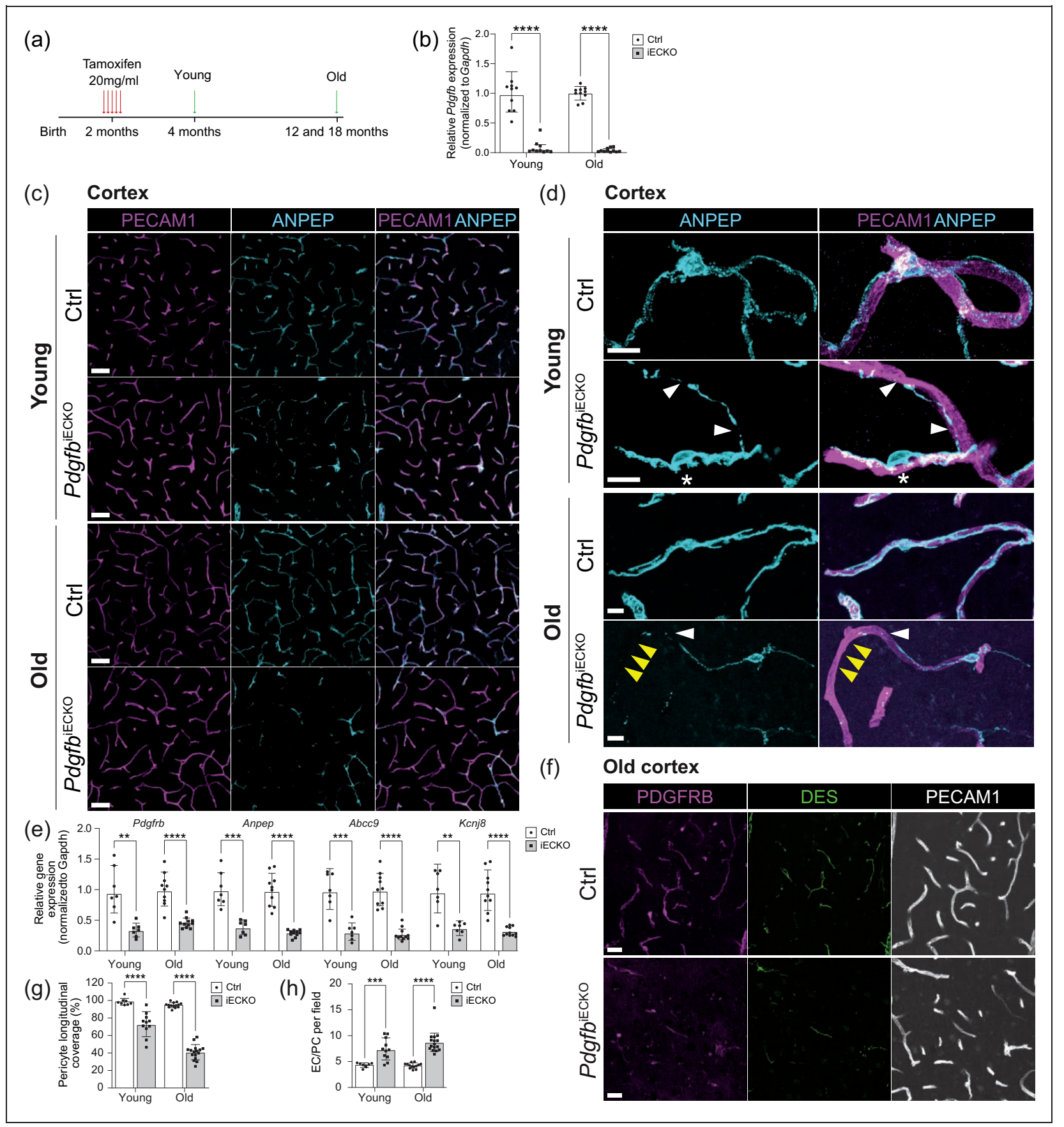

Figure I. Experimental scheme, gene deletion efficiencies and assessment of pericytes in adult-induced Pdgf $b^{\mathrm{iECKO}}$ and littermate controls. (a) Endothelial-specific Pdgfb deletion was accomplished by Tamoxifen administration for 5 days at 2 months of age. All analyzes were performed at 4 months of age for the "young" age and at 12- and I8-months for the "old" age. (b) qPCR analysis of Pdgfb mRNA expression on freshly isolated brain microvascular fragments. Pdgfb mRNA expression was normalized to endogenous Gapdh levels. In young mice, $8 \%$ of Pdgfb expression remained in Pdgfb ${ }^{\mathrm{iECKO}}$ mice $(\mathrm{n}=10)$. In old mice, $4 \%$ of $P d g f b$ expression remained in $P d g f b^{\mathrm{iECKO}}$ mice $\left(P d g f b^{\mathrm{iECKO}} \mathrm{n}=1 \mathrm{I}, \mathrm{Ctrl} \mathrm{n}=10\right)$. (c) Representative overview images of mural cells from the cortex of young and old mice. Co-immunolabeling of PECAMI (magenta) and ANPEP (cyan). Scale bars $50 \mu m$. (d) Representative high magnification images to visualize pericyte morphology in young and old Pdgf $b^{\mathrm{iECKO}}$ and controls. Co-immunolabeling of PECAMI (magenta) and ANPEP (cyan). White arrowheads indicate fragmented pericyte processes. Asterisk indicates pericytes with altered cell bodies and distinct foot processes in Pdgfb ${ }^{\mathrm{iECKO}}$. Yellow arrowheads indicate shorter processes leaving part of the vasculature uncovered in Pdgfb ${ }^{\mathrm{iECKO}}$. Scale bars $10 \mu \mathrm{m}$. (e) qPCR analysis on the mural cell genes Pdgfrb and Anpep and the pericyte genes Abcc9 and Kcnj8 performed on freshly isolated brain microvascular fragments from young and old mice (for litter and $n$ number see Supplementary Table I). The genes of interest were normalized to endogenous Gapdh levels and are presented as relative gene 


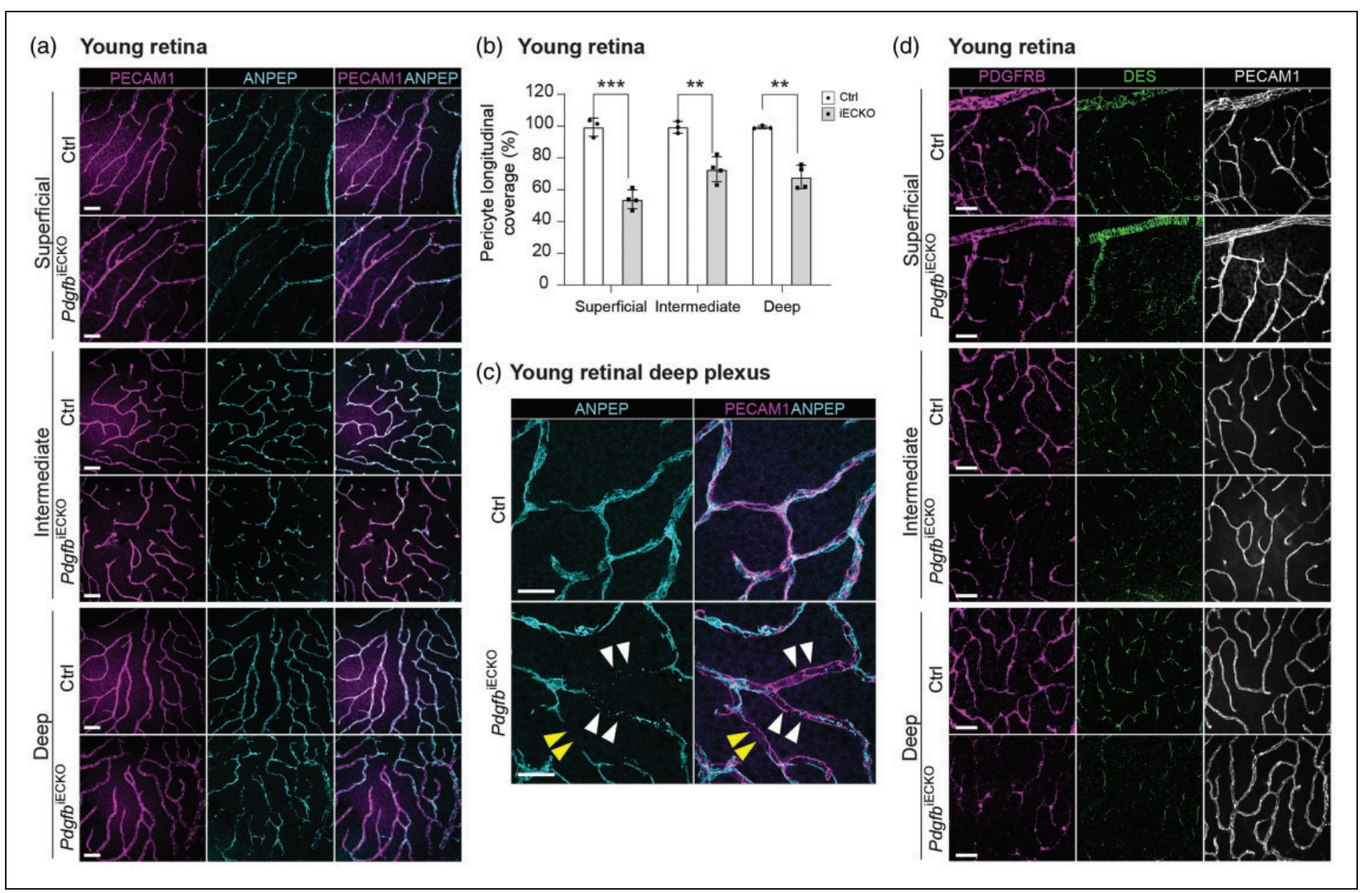

Figure 2. Assessment of pericytes in adult-induced $P d g f b^{\mathrm{iECKO}}$ and littermate control retinas (a) Representative overview images of mural cells from the retinal plexuses of young mice. Co-immunolabeling of PECAMI (magenta) and ANPEP (cyan). Scale bars $50 \mu \mathrm{m}$. (b) The skeletal length of PECAMI positive capillaries and ANPEP positive pericytes in Pdgfb $b^{\text {iECKO }}$ and control plexuses were measured and plotted as the percentage of pericyte longitudinal length over blood vessel length $\left(P d g f b^{\mathrm{iECKO}} \mathrm{n}=4, \mathrm{Ctrl} n=3\right)$. (c) Representative high magnification images to visualize pericyte morphology in young Pdgf $b^{\mathrm{iECKO}}$ and controls. Co-immunolabeling of PECAMI (magenta) and ANPEP (cyan). White arrowheads indicate fragmented pericyte processes. Yellow arrowheads indicate shorter processes leaving part of the vasculature uncovered in Pdgfb $b^{\mathrm{iECKO}}$. Scale bars $25 \mu \mathrm{m}$. (d) Representative overview images from young mice. Co-immunolabeling of PDGFRB (magenta), DES (green) and PECAMI (white). Scale bars $50 \mu \mathrm{m}$.

vascular phenotype observed in mouse models with developmental pericyte loss. ${ }^{6,7,10,15}$ There was also no regional difference in pericyte coverage, in $\mathrm{EC} / \mathrm{PC}$ ratio or EC number per field (Suppl. Fig. 2E, F, G), however there was a reduction in vascular density in the cortex of 12-month-old $P d g f b^{\text {iECKO }}$ mice when compared to controls (Suppl. Fig. 2H).
Similar to brain, retinal vasculature showed decrease in longitudinal coverage of ANPEP-positive pericytes in all three vascular plexuses in 4-month-old mice (Figure 2(a)). The largest loss was observed and quantified in superficial vascular plexus $(54 \%$ coverage remaining), whereas the intermediate and deep plexuses showed a lower degree of pericyte loss (73 and 71\%

Figure I. Continued.

expression to Ctrl samples. (f) Representative overview images from the cortex of old mice. Co-immunolabeling of PDGFRB (magenta), DES (green) and PECAMI (white). Scale bars $25 \mu \mathrm{m}$. (g) The skeletal length of PECAMI positive capillaries and ANPEP positive pericytes in Pdgf ${ }^{\mathrm{iECKO}}$ and controls was measured and plotted as the percentage of pericyte longitudinal length over blood vessel length. Three litters were analyzed for pericyte coverage in the cortex of young mice $\left(P d g b^{\mathrm{iECKO}} \mathrm{n}=\mathrm{II}, \mathrm{Ctrl} \mathrm{n}=8\right)$ and five litters were analyzed for coverage in the cortex of old mice $\left(P d g f b^{i E C K O} n=16, C \operatorname{trl} n=13\right)$. (h) Quantification of endothelial cell $\left(\mathrm{ERG}^{+}\right)$to pericyte $\left(\mathrm{ANPEP}^{+}, \mathrm{DAPI}^{+}\right)$ratio per field in young $\left(P d g f b^{\mathrm{iECKO}} \mathrm{n}=1 \mathrm{I}, \mathrm{Ctrl} \mathrm{n}=8\right)$ and old mice $\left(P d g f b^{\mathrm{iECKO}} \mathrm{n}=16, \mathrm{Ctrl}\right.$ $\mathrm{n}=13$ ). b, $\mathrm{g}$ and $\mathrm{h}$-Old, normality tests revealed that the data was unevenly distributed so nonparametric Mann-Whitney $U$ test was used to evaluate significance. e and h-Young, the significance of evenly distributed data was evaluated using unpaired 2-tailed $t$ test with Welch's correction. e, Gene expression comparison between young and old Pdgf $b^{\mathrm{iECKO}}$ mice was not significant for neither of the four genes. Data is presented as geometric mean with geometric SD. $* * p<0.01$, $* * * p=0.00$ I, $* * * * p<0.000$ I, ns $=$ not significant. 
coverage remaining, respectively), which was similar in magnitude to the brain (Figure 2(b)). The total length of retinal vasculature (Suppl. Fig. 2I) and total ERG+ endothelial cell numbers per field were comparable in mutants and controls in all three plexuses (Suppl. Fig. 2J). Observation at high magnification revealed that pericyte processes in retinas of $P d g f b^{\text {iECKO }}$ mice were thinner, partly detached and displayed discontinuous ANPEP staining (Figure 2(c)), which resembled the mutant brain pericyte phenotype. Also the pericyte markers DES and PDGFRB were reduced in the 4-months-old retinas (Figure 2(d)). Analysis of 18-month-old control $(\mathrm{n}=1)$ and $P d g f b^{\text {iECKO }}(\mathrm{n}=2)$ retinas showed further loss of pericyte coverage without any obvious vascular dilation when compared to 4-month-old retinas (Suppl. Fig. $2 \mathrm{~K}$ ), again similar to the observations in brain.

Next, the $P d g f b^{\text {iECKO }}$ mice were analyzed 2 weeks after tamoxifen induction (Suppl. Fig. 3 A) in order to examine whether there was any pericyte loss shortly after $P d g f b$ gene deletion, and if pericyte death could be recorded. Anti-ANPEP immunostaining showed a slight $(12 \%)$ but significant decrease in pericyte longitudinal coverage (Suppl. Fig. 3B, white arrowhead, C). However, there was no significant difference in EC/PC ratio (Suppl. Fig. 3D), vascular length (Suppl. Fig. 3E) or total number of ERG+ endothelial cells per field (Suppl. Fig. 3F). Staining for the apoptotic cell marker Cleaved-CASPASE3 showed that very few cells were undergoing apoptosis at this time in both $P d g f b^{\text {iECKO }}$ and littermate control brains (Suppl. Fig. 3H). A small number of these were positive for PECAM1, indicating that they were endothelial cells (Suppl. Fig. 3G, white asterisk and $\mathrm{H}$ ), and even fewer were perivascular cells (Suppl. Fig. 3G, white arrowhead and $\mathrm{H}$ ). These observations suggest that PDGFB is important for the maintenance of pericyte coverage during vascular homeostasis in the adult brain, and further that pericyte degeneration following $P d g f b$ ablation is a slow process. While we failed to observe increased apoptotic pericytes by staining for Cleaved-CASPASE3, we cannot exclude the possibility that pericyte apoptosis takes place but remains too asynchronous and/or fast to be captured using immunohistochemical detection.

In contrast to pericyte coverage, arteriolar VSMC coverage was only moderately affected in $P d g f b^{\text {iECKO }}$ mice when compared to littermate controls (Figure 3(a) to (c)). Immunofluorescence staining with ACTA2 antibodies showed even vascular coverage by VSMC in brain and retina of control mice, while it was slightly irregular in shape and sparser in the $P d g f b^{\text {iECKO }}$ mice (Figure 3(a) and (b)). More specifically, there was an occasional patchy loss of expression of ACTA2 in $P d g f b^{\text {iECKO }}$ mice, leaving gaps in the ACTA2 staining, similar to but less extensive than what has previously be reported for Notch3 knockouts ${ }^{27}$ (Figure 3(a) and (b), white arrows). Upon closer examination of both brain and retina, we found that while ACTA2 staining terminates bluntly at points of arteriolar-capillary transition in control mice (Figure 3(a) and (b), yellow arrowheads) the VSMCs stretched out and assumed an elongated pericyte-like morphology at these sites in $P d g f b^{\text {iECKO }}$ mice (Figure 3(a) and (b), white arrowheads). qPCR of the VSMC markers Acta2 and Tagln showed a significant reduction in brains of old mice (Acta2 (61\% remaining) and Tagln (55\% remaining)), whereas the difference was not significant in young mice (Figure 3(c)).

\section{Effects of adult loss of pericytes on the mouse brain vasculature}

Previous analyses have shown that developmental loss of pericytes, as occurs in constitutive $P d g f b$ mutants including $P d g f b^{\text {ret/ret }}$ and other adult-viable $P d g f b$ loss-of-function mouse models, has a number of different consequences for brain vascular structure and function. ${ }^{9,10,13-15,28,29}$ These include the formation of a sparser network of wider capillaries, the specific reduction in the expression of certain BBB transporters, upregulated endothelial expression of growth factors and pro-inflammatory molecules, activation of angiogenic sprouting, the occurrence of two distinct modes of BBB impairment, i.e. increased transcytosis and hotspot leakage via disturbed endothelial junctions, and the progressive formation of microvascular-associated calcifications in deep brain regions modeling the human genetic disease PFBC. Although all of the mentioned abnormalities have been documented in adult $P d g f b^{\text {ret/ret }}$ mice, it is not clear whether some or all of them reflect defective vascular development rather than a role for pericytes in adult vascular homeostasis. We therefore studied the vascular consequences of adultinducible pericyte loss in $P d g f b^{\text {iECKO }}$ mice. As shown above (Figures 1 to 3 ), the general capillary rarefaction and dilation, which hallmarks the $P d g f b^{\text {ret/ret }}$ brain vasculature, were not reproduced in $P d g f b^{\text {iECKO }}$ mice, and they therefore likely reflect a critical role for pericytes during developmental brain vascular morphogenesis.

We next analyzed the expression of the capillaryvenous-specific transporters transferrin receptor $\mathrm{C}$ (TFRC) and solute carrier family 16 member 1 (SLC16A1), which are both strongly downregulated in $P d g f b^{\text {ret/ret }}$ mouse brain vasculature. ${ }^{15}$ Here, ACE2 antibody was used for visualizing the pericytes. ${ }^{18} \mathrm{We}$ found that both transporters retained normal levels and distribution of expression in $P d g f b^{\text {iECKO }}$ capillaries and venules (negative in arterioles, Figure 4(a) and (b), asterisks) devoid of pericytes even in old mice 


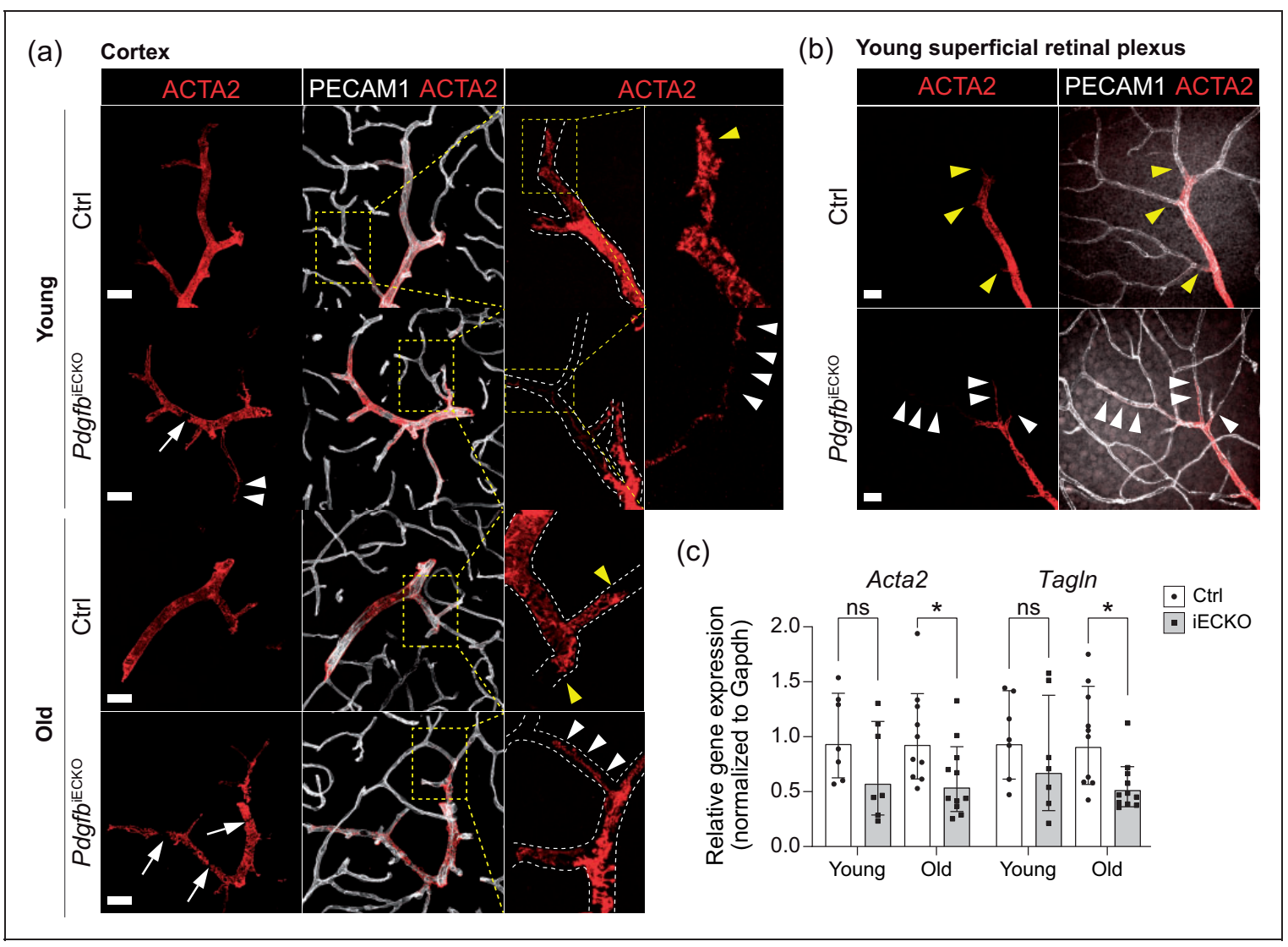

Figure 3. Assessment of arteriolar VSMCs (aaVSMCs) in adult-induced Pdgf $b^{\mathrm{iECKO}}$ and littermate controls. (a) Representative images of aaVSMC in young (Pdgfb $\left.b^{\mathrm{iECKO}} \mathrm{n}=4, \mathrm{Ctrl} n=2\right)$ and old mice $(\mathrm{n}=3)$ : co-immunolabeling of ACTA2 (red) and PECAMI (white). Scale bars $30 \mu \mathrm{m}$. Dashed inserts are magnified for detailed visualization of ACTA2 expression and aaVSMC morphology. (b) Representative images of aaVSMC in young retinas $\left(P d g f b^{i E C K O} n=4, C \operatorname{trl} n=3\right.$ ): co-immunolabeling of ACTA2 (red) and PECAMI (white). Scale bars $25 \mu \mathrm{m}$. In a and b, white arrows indicate patchy loss of ACTA2 expression leaving small, empty gaps of aaVSMC coverage along the vasculature. Yellow arrowheads indicate ACTA2 positive terminal aaVSMC at the branching arterioles in Ctrl samples, where they appeared to end sharply while in Pdgf $b^{\mathrm{iECKO}}$ they were stretched out and elongated (white arrowheads). (c) qPCR analysis on the mural cell genes Acta2 and Tagln performed on freshly isolated brain microvascular fragments (for litter and $\mathrm{n}$ number see Supplementary Table I). The genes of interest were normalized to endogenous Gapdh levels and they are presented as relative gene expression to Ctrl samples. c-Old (Tagln), Normality tests revealed that the data was unevenly distributed so nonparametric Mann-Whitney $U$ test was used to evaluate significance. c-Young (Acta2, Tagln) and c-Old (Acta2), The significance of evenly distributed data was evaluated using unpaired 2-tailed $t$ test with Welch's correction. ${ }^{*} p<0.05, * * p<0.0$ I, $*^{* * *} p<0.00 \mathrm{I}$ and $*_{* * * *} p<0.000$ I, two-tailed student's t-test. Data is presented as geometric mean with geometric SD.

(Figure 4(a) and (b), white arrowheads). This suggests that there is also no change in the endothelial arteriovenous zonation in $P d g f b^{\mathrm{iECKO}}$, although more detailed analyses of endothelial gene expression will be required for a definitive conclusion. Also, adherens (CDH5) and tight (CLDN5) junctions showed overall normal expression patterns in vascular stretches devoid of pericytes in $P d g f b^{\mathrm{iECKO}}$ mice (Figure 4(c)), which is similar to the overall picture of $P d g f b^{\text {ret/ret }}$ mouse brain vasculature. ${ }^{15}$ However, we found that mRNA levels for three growth factors/regulators, Fgfbpl, Bmp6 and Angpt2, which are all strongly upregulated in
$P d g f b^{\text {ret/ret }}$ brain endothelial cells, ${ }^{15}$ were also upregulated in the $P d g f b^{\text {iECKO }}$ brain endothelium compared with littermate controls at both young and old age (Figure 4(d)). Immunofluorescence stainings further showed that capillary stretches without pericyte contact had increased ANGPT2 expression both in cortex (Figure 4(e)) (white arrowheads and inset) and hippocampus (Figure 4(f)) (white arrowheads and inset ii) in old $P d g f b^{\text {iECKO }}$ brains. We were not able to confirm the increased ANGPT2 expression by immunofluorescence in young $P d g f b^{\text {iECKO }}$ brains. On the other hand, leukocyte adhesion molecule, ICAM1 protein, was 


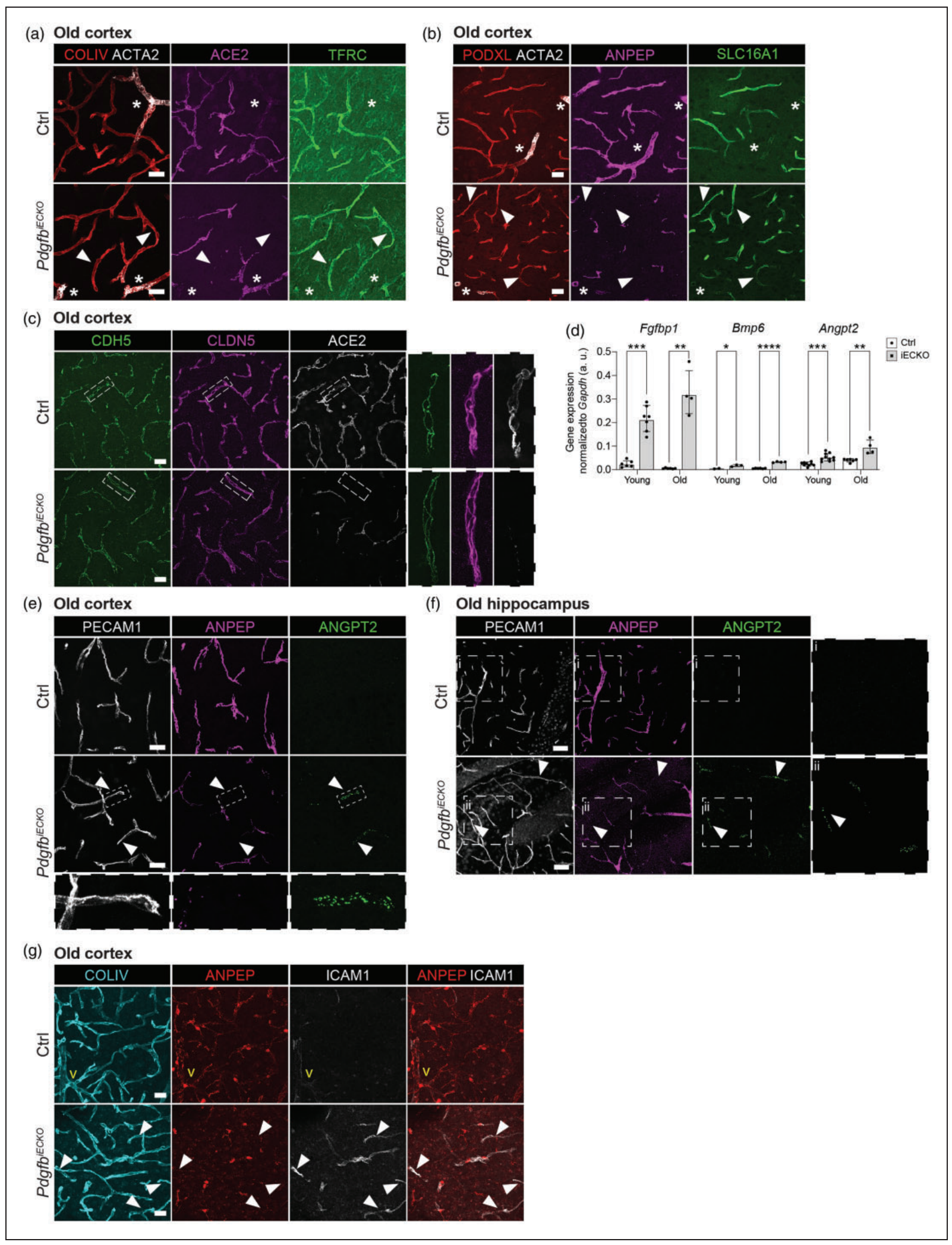

Figure 4. Characterization of endothelial cell response to pericyte loss in adult-induced $P d g f b^{\mathrm{iECKO}}$. (a) Representative images from the cortex of old Pdgfb $b^{\mathrm{iECKO}}(\mathrm{n}=7)$ and littermate controls $(n=6)$. Co-immunolabeling of COLIV (red), ACTA2 (white), ACE2 (magenta) and TFRC (green). Scale bars $25 \mu \mathrm{m}$. (b) Representative images from the cortex of old Pdgfb $b^{\text {iECKO }}(\mathrm{n}=7)$ and littermate controls $(n=6)$. Co-immunolabeling of PODXL (red), ACTA2 (white), ANPEP (magenta) and SLCI6AI (green). Arrowheads mark 
upregulated in capillaries of both young and old brains and young retinas (Figure 4(g) and Suppl. Fig. 4).

$P d g f b$ loss-of-function models with developmental pericyte hypoplasia show increased BBB permeability for different molecular weight tracers, which correlates with the degree of pericyte-deficiency in the particular model. ${ }^{10,15}$ In order to assess whether adult-induced pericyte loss causes vascular permeability in $P d g f b^{\mathrm{iECKO}}$ mice, we measured intravenously injected Alexa Fluor 555 Cadaverine $(1 \mathrm{kDa})$ accumulation in the brain after $2 \mathrm{~h}$ circulation, a protocol that readily detects BBB leakage in $P d g f b^{\text {ret/ret }}$ mice (with $25 \%$ longitudinal pericyte coverage). ${ }^{10,15}$ Using this protocol, we detected a significant increase in BBB permeability in old $P d g f b^{\text {iECKO }}$ mice compared to their littermate controls (Figure 5(a)). Although we noticed a similar trend also in young $P d g f b^{\text {iECKO }}$ mice, this difference was not statistically significant (Figure 5(a)). We could not detect Alexa Fluor 555 Cadaverine leakage in any of the 4-month-old retinal plexuses (Figure 5 (b)). To assess if hotspot BBB leakage sites occurred in old $P d g f b^{\text {iECKO }}$ mice similar to the situation in $P d g f b^{\text {ret/ret }}$ mice, we systemically injected $70 \mathrm{kDa}$ tetramethylrhodamine-dextran (TMR-dextran) and analyzed the mice after $16 \mathrm{~h}$ of tracer circulation. Macroscopical fluorescence analysis did not reveal any increased permeability (Figure 5(c)), unlike the situation in $P d g f b^{\text {ret/ret }}$ mice. ${ }^{15}$ However, by assessing the presence of extravascular TMR-dextran hotspots in the brain sections together with endothelial and pericyte markers (PECAM1 and ANPEP, respectively), we detected hotspots in the different regions of old $P d g f b^{\text {iECKO }}$ mouse brains (Figure 5(d)) albeit at lower abundance compared to $P d g f b^{\text {ret/ret }}$ mice. ${ }^{15}$ Vascular stretches associated with TMR-dextran leakage were invariably dilated and devoid of pericytes (Figure 5 (d)) and surrounded by activated microglia (Figure 5 (e)). We found approximately 4 hotspots per section in the $P d g f b^{\text {iECKO }}$ mice while $<1$ was detected in the controls (Figure 5(f)). For comparison, $P d g f b^{\text {ret/ret }}$ brains displayed approximately 50 hotspots per section. ${ }^{15}$ As in $P d g f b^{\text {ret/ret }}$ brains, the TMR-dextran hotspots in $P d g f b^{\text {iECKO }}$ mouse brains showed abnormal distribution of the junctional proteins CDH5 and CLDN5 (Figure 5(g)), decreased SLC16A1 and TFRC expression (Figure 5(h)), increased ICAM1 expression (Figure 5(1)) and undetectable ANGPT2 (Figure 5(j)). Taken together, we observed similar, albeit less extensive, BBB abnormalities in old $P d g f b^{\mathrm{iECKO}}$ mouse brains as in $P d g f b^{\text {ret } / \text { ret }}$ mice.

Finally, since mice hypomorphic for $P d g f b$ and humans haploinsufficient for $P D G F B$ develop vesselassociated calcifications in the brain, ${ }^{9}$ we asked if adult-onset deletion of $P d g f b$ triggers capillary calcification. We performed immunohistochemical analysis of vessel-associated calcifications by staining for three different proteins that selectively accumulate in calcifications, amyloid precursor protein (APP), amyloid precursor like protein-2 (APLP2) and osteopontin $(\mathrm{SPP} 1)^{13,28}$ in old control and $P d g f b^{\mathrm{iECKO}}$ mice. Four to six $75 \mu \mathrm{m}$-thick sagittal sections were analyzed per mouse, with sections chosen to include both outer cortex and deep brain regions in the analysis, the latter known to harbor abundant calcifications in $P d g f b^{\text {ret/ret }}$ mice. In altogether $16 P d g f b^{\text {iECKO }}$ and 12 control mice, we detected only a single calcification in one of the 12-months-old $P d g f b^{\text {iECKO }}$ mice (Suppl. Fig. 5A). Analysis of two additional 12-months-old control and $P d g f b^{\text {iECKO }}$ mice using various histochemical stains, including Alizarin Red, did not reveal any signs of vessel-associated calcification (data not shown). Presence of osteopontin (SPP1) has been noticed in small non-calcified vessel-associated nodules in $P d g f b^{\text {ret/ret }}$ brain thalamus and midbrain regions. ${ }^{28}$ While we could identify rare small SPP1 positive nodules associated with the vasculature in the thalamus

\section{Figure 4. Continued.}

capillaries without pericyte contact. Asterisks mark ACTA2+ arteries/arterioles. Scale bars $25 \mu \mathrm{m}$. (c) Representative images from the cortex of old Pdgfb ${ }^{\mathrm{iECKO}}(\mathrm{n}=7)$ and littermate controls $(\mathrm{n}=6)$. Co-immunolabeling of CDH5 (green), CLDN5 (magenta) and ACE2 (white) positive pericytes. Scale bars $25 \mu \mathrm{m}$. Dashed insets are magnified for detailed visualization of junctions. (d) qPCR analysis on the endothelial cell genes Fgfbpl, Bmp6 and Angpt2 performed on freshly isolated brain microvascular fragments in young and old mice (for litter and $\mathrm{n}$ number see Supplementary Table I). The genes of interest were normalized to endogenous Gapdh levels ( $\Delta \mathrm{Cq})$ and these are presented as arbitrary units (a. u.). For old (Angpt2), normality tests revealed that the data was unevenly distributed so nonparametric Mann-Whitney $U$ test was used to evaluate significance. For young (Angpt2, Fgfbpl and Bmp6) and old (Fgfbpl and $B m p 6)$, the significance of evenly distributed data was evaluated using unpaired 2-tailed $t$ test with Welch's correction. $* p<0.05$, ${ }^{* *} p<0.01$, $*^{* *} p<0.001$ and ${ }^{* * * *} p<0.000$ I. Data is presented as geometric mean with geometric SD. (e, f) Representative images from the cortex $(E)$ and hippocampus $(F)$ of old $P d g b^{\mathrm{iECKO}}$ and littermate controls. Co-immunolabeling of PECAMI (white) and ANGPT2 (green) and ANPEP (magenta). Arrowheads mark capillaries with ANGPT2 expression and without pericyte contact. In (e) dashed inset shows a magnified ANGPT2+ vessel lacking ANPEP expression in the Pdgf $b^{\text {iECKO } . ~} \mathrm{n}=9$, scale bar $10 \mu \mathrm{m}$. In $(\mathrm{f})$,

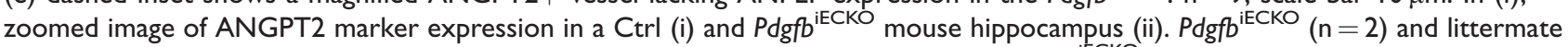
controls $(n=3)$. Scale bars $50 \mu \mathrm{m}$. (g) Representative images from the cortex of old Pdgfb $b^{\mathrm{iECKO}}(\mathrm{n}=10)$ and littermate controls $(n=9)$. Co-immunolabeling of COLIV (cyan) and ANPEP (red) and ICAMI (white). Arrowheads mark capillaries with ICAMI expression and without pericyte contact. In yellow, the vein is marked as " $v$ ". Scale bars $25 \mu \mathrm{m}$. 


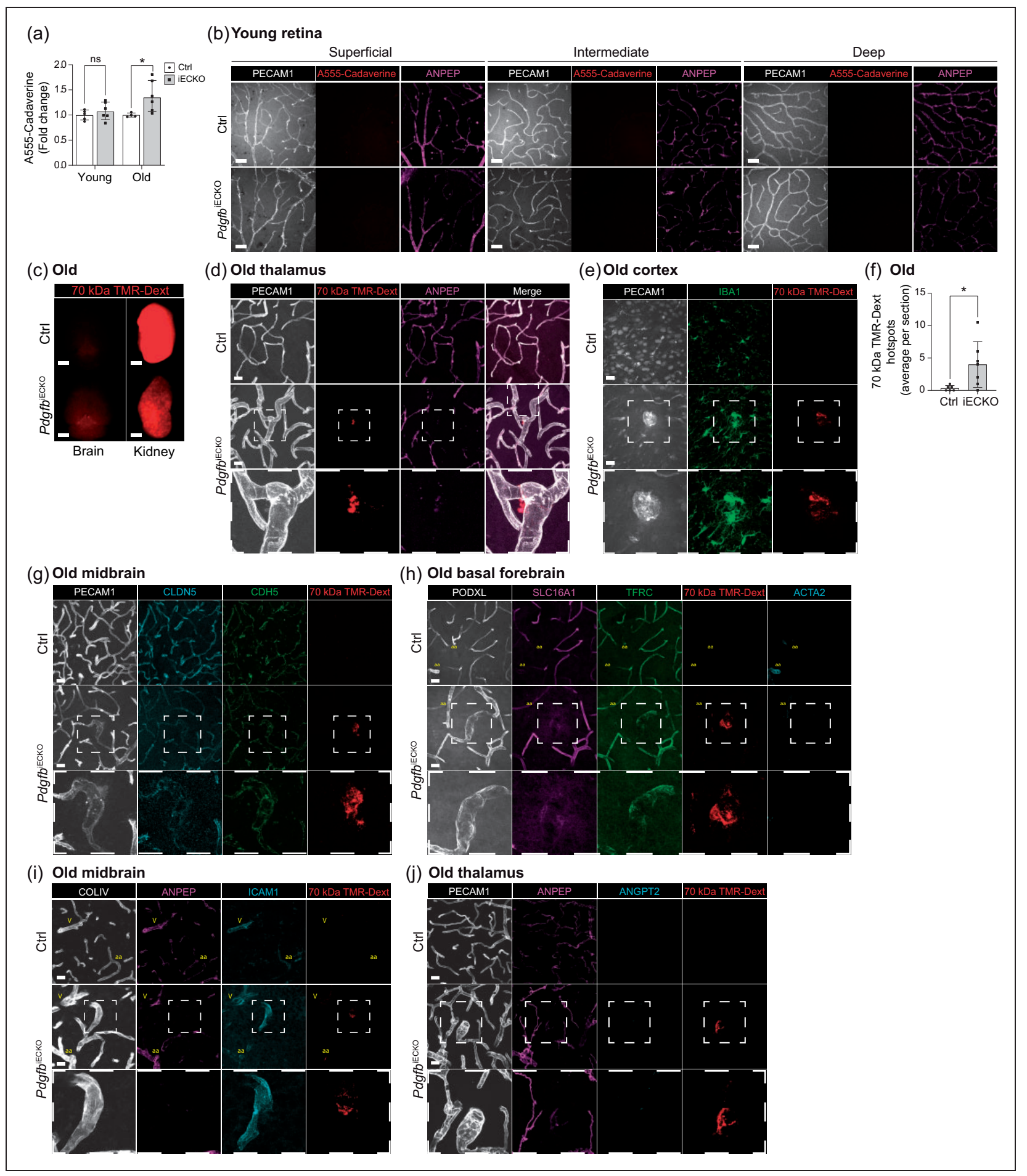

Figure 5. Analysis of blood-brain and blood-retina barrier permeability in adult-induced $P d g f b^{\mathrm{iECKO}}$ compared to littermate controls. (a) Quantification of I kDa Alexa Fluor 555 cadaverine permeability in young ( 2 independent experiments, Pdgf $b^{\mathrm{iECKO}} \mathrm{n}=6, \mathrm{Ctrl}$ $\mathrm{n}=5$ ) and old ( 2 independent experiments $P d g f b^{\mathrm{iECKO}} \mathrm{n}=6, C \operatorname{trl} \mathrm{n}=3$ ) mice after $2 \mathrm{~h}$ circulation. Y-axis shows the fold change in permeability in relation to controls. The significance was evaluated using unpaired 2-tailed $t$ test with Welch's correction. $* p<0.05$, $\mathrm{ns}=$ not significant. Data is presented as geometric mean with geometric SD. (b) Representative images of I kDa Alexa Fluor 555 cadaverine tracer permeability in young $\left(P d g f b^{\mathrm{iECKO}} \mathrm{n}=4, \mathrm{Ctrl} n=3\right)$ retinal plexuses after $2 \mathrm{~h}$ circulation. Co-immunolabeling of PECAMI (white), ANPEP (magenta), tracer (red) did not show any vascular leakage. Scale bars $50 \mu \mathrm{m}$. (c) Representative images of whole brains and kidneys after overnight circulation of $70 \mathrm{kDa}$ TMR-dextran in old mice (two litters, Pdgfb ${ }^{\mathrm{iECKO}} \mathrm{n}=7, \mathrm{Ctrl} \mathrm{n}=6$ ). Scale bars $2 \mathrm{~mm}$. (d) Representative images of the leakage in old mice. Co-immunolabeling of PECAMI (white), ANPEP (magenta), 
and midbrain regions, these were found both in control and $P d g f b^{\text {iECKO }}$ brains (Suppl. Fig. 5B). We also could not detect any calcifications at hotspot leakage sites (Suppl. Fig. 5C). Based on these observations, we conclude that loss of $P d g f b$ must take place during development in order for vascular calcifications to develop, at least at a size and abundance detectable by our current protocols.

\section{Discussion}

It is well established that endothelial-derived PDGFB is crucial for pericyte recruitment during embryonic and early postnatal brain vascular development, an effect mediated by stimulation of pericyte proliferation and migration via PDGFRB. ${ }^{4-7,20,21}$ Adult-viable constitutive $P d g f b$ loss-of-function mutants with strongly reduced pericyte coverage acquire abnormal brain vasculature including general microvessel dilation and rarefaction, skewed endothelial arterio-venous zonation, reduced expression of specific transporters, two models of BBB leakage, and regional microvascular calcification. ${ }^{9,10,13-15,28,29}$ In this study, using an adult-inducible model of $P d g f b$ ablation, we demonstrate that endothelial-derived PDGFB is also required for adult maintenance of pericyte coverage, but that the slowly progressing pericyte loss that follows upon adult-induced $P d g f b$ ablation causes some, but not all, of the abnormalities observed in constitutive $P d g f b$ mutants.

The partially different consequences of constitutive or inducible $P d g f b$ ablation raises questions about the importance of the developmental state during which $P d g f b$ ablation takes place, as well as the extent of ablation. In general, it is difficult to obtain complete gene deletion in adult tissues using tamoxifen-inducible strategies. We have previously shown that the constitutive absence of one functional $P d g f b$ allele, leading to a $50 \%$ loss of $P d g f b$ transcripts, is not sufficient to render a significant alteration in pericyte density in the brain vasculature. ${ }^{25}$ Apparently, a threshold level of $P d g f b$ expression exists below (or at) $50 \%$ of normal, which is sufficient for the maintenance of normal pericyte numbers. Park et al. reported that deletion of $P d g f b$ using $C d h 5$-CreERT2 at 8-weeks of age followed by analysis at 12 -weeks of age did not lead to altered pericyte morphology or coverage in the mouse retina. ${ }^{22}$ The difference to our current findings reflects that a longer time of $P d g f b$ depression was needed to cause pericyte loss also in the retina. A second possibility is that a sufficiently high gene deletion efficiency was not obtained - this was not assessed in the study. ${ }^{22}$

In our own study, $>90 \%$ loss of endotheliumderived $P d g f b$ at 2 months of age resulted in $54-74 \%$ (in 4-moths-old retina and brain) and 43\% (in 12- and 18-months-old brain) remaining longitudinal pericyte coverage compared with controls. Residual pericytes further displayed abnormal phenotypes in that their cell bodies were frequently rounded and partially detached from the endothelium, and their processes appeared thinner, partially detached and with altered secondary foot processes than in controls. With these observations, we propose that endothelium-derived $P d g f b$ is necessary for the maintenance of a normal pericyte phenotype, as well as for long term maintenance of normal pericyte numbers.

Although we detected changes in arteriolar VSMC morphology and coverage in $P d g f b^{\mathrm{iECKO}}$ mice, brain and retinal arteriolar VSMC appeared less sensitive to $P d g f b$ loss than pericytes. A similar discrepancy

Figure 5. Continued.

$70 \mathrm{kDa}$ TMR-dextran (red) in thalamus. Dashed inset shows a $70 \mathrm{kDa}$ TMR-dextran hotspot on an enlarged capillary lacking pericyte contact (two litters, Pdgfb $b^{\mathrm{iECKO}} \mathrm{n}=7, \mathrm{Ctrl} \mathrm{n}=6$ ). Scale bars $25 \mu \mathrm{m}$. (e) Representative images of the cerebral cortex of old mice. Co-immunolabeling of PECAMI (white), IBAI (green) and $70 \mathrm{kDa}$ TMR-dextran (red). Dashed inset shows a $70 \mathrm{kDa}$ TMR-dextran hotspot on an enlarged capillary surrounded by activated microglia (IBAI+ cells) (two litters, Pdgfb $\left.{ }^{\mathrm{iECKO}} \mathrm{n}=7, \mathrm{Ctrl} n=6\right)$. Scale bars $25 \mu \mathrm{m}$. (f) Quantification of extravascular $70 \mathrm{kDa}$ TMR-Dextran hotspots in old mice (two litters, Pdgfb ${ }^{\mathrm{iECKO}} \mathrm{n}=7, \mathrm{Ctrl} \mathrm{n}=6$ ). Y-axis shows the average number of hotspots detected per brain sections from each mouse. The significance was evaluated using unpaired 2 -tailed $t$ test with Welch's correction. $*_{p}<0.05$. Data is presented as mean $\pm S D$. (g) Representative images of the midbrain of aged mice. Co-immunolabeling of PECAMI (white), CLDN5 (cyan), CDH5 (green) and 70 kDa TMR-dextran (red). Dashed inset shows a $70 \mathrm{kDa}$ TMR-dextran hotspot on an enlarged capillary with disturbed junctional expression (two litters, Pdgf $b^{\mathrm{iECKO}} \mathrm{n}=7, \mathrm{Ctrl} \mathrm{n}=6$ ). Scale bars $25 \mu \mathrm{m}$. (h) Representative images of the basal forebrain of old mice. Co-immunolabeling of PODXL (white), SLCI6AI (magenta), TFRC (green), ACTA2 (cyan), and 70 kDa TMR-dextran (red). The arterioles are marked as "aa" in yellow. Dashed inset shows a $70 \mathrm{kDa}$ TMR-dextran hotspot on an enlarged capillary with decreased expression of the endothelial proteins SLCI6AI and TFRC (two litters, Pdgfb ${ }^{\mathrm{iECKO}} \mathrm{n}=7, \mathrm{Ctrl} \mathrm{n}=6$ ). Scale bars $25 \mu \mathrm{m}$. (i) Representative images of the midbrain of old mice. Co-immunolabeling of COLIV (White), ANPEP (magenta), ICAMI (cyan) and $70 \mathrm{kDa}$ TMR-dextran (red). In yellow, the arterioles are marked as "aa" and the veins as " $v$ ". Dashed inset shows a $70 \mathrm{kDa}$ TMR-dextran hotspot on an enlarged capillary lacking pericyte contact with unusual expression of ICAMI (two litters, Pdgf ${ }^{\mathrm{iECKO}} \mathrm{n}=7, \mathrm{Ctrl} \mathrm{n}=6$ ). Scale bars $25 \mu \mathrm{m}$. (j) Representative images of the thalamus of old mice. Co-immunolabeling of COLIV (White), ANPEP (magenta), ANGPT2 (cyan) and 70 kDa TMR-dextran (red). Dashed inset shows a $70 \mathrm{kDa}$ TMR-dextran hotspot on an enlarged capillary lacking pericyte contact with no expression of ANGPT2 (two litters, Pdgfbi ${ }^{\mathrm{iECKO}} \mathrm{n}=7, \mathrm{Ctrl} \mathrm{n}=6$ ). Scale bars $25 \mu \mathrm{m}$. 
was observed in constitutive $P d g f b$ or $P d g f r b$ knockout mutants, which showed residual arteriolar VSMC coverage in spite of strongly reduced number of pericytes. $^{4,5}$ Of note, brain arterial VSMC also express significant levels of $P d g f d$, which encodes PDGFD, another PDGF ligand with affinity for PDGFRB $^{18}$ thus possibly compensating for the loss of paracrine PDGFB from endothelial cells.

Because $P d g f b^{\text {ret/ret }}$ mice display two distinct modes of BBB permeability - increased transcytosis and focal hotspot leakage, ${ }^{10,15}$ we were interested to know how inducible adult pericyte loss affects BBB permeability. We found that 4-months-old $P d g f b^{\text {iECKO }}$ mouse retinas and brains with $54-74 \%$ longitudinal pericyte coverage did not display significantly increased leakage of Alexa Fluor 555 cadaverine while 12-18-months-old $P d g f b^{\text {iECKO }}$ mice brains with $43 \%$ longitudinal pericyte coverage did. We have previously demonstrated that adult-viable $P d g f b$ single allele rescue mice $\left(\mathrm{R} 26 \mathrm{P}^{+/ 0}\right)$ with $40 \%$ pericyte coverage showed a leaky BBB, whereas $P d g f b$ double allele rescue mice $\left(\mathrm{R} 26 \mathrm{P}^{+/+}\right)$ with $72 \%$ pericyte coverage did not. ${ }^{10}$ Thus, the inducible and constitutive mutants suggest a similar threshold level of pericyte coverage $(>50 \%)$ beyond which further pericyte loss causes BBB disruption. Interestingly, $>50 \%$ pericyte loss also correlated with the development of proliferative retinopathy in endothelial-specific constitutive $P d g f b$ knockouts. ${ }^{20}$ Others have reported that $20 \%$ reduced pericyte coverage disrupts the $\mathrm{BBB},{ }^{30}$ an observation that we cannot confirm using our adult-inducible $P d g f b$ knockout and methods for BBB leakage measurements.

Developmental loss of pericytes results in skewed vascular arterio-venous zonation. ${ }^{15}$ When analyzing the capillary-venous marker, TFRC and SLC16A1, expression in old $P d g f b^{\text {iECKO }}$ brain vasculature, we could not detect any general loss of their expression in capillary stretches devoid of pericytes. However, the hotspot leakage sites had lost or reduced expression of SLC16A1 and TFRC. Similar to the situation for developmental loss of pericytes, adherens and tight junction protein expression pattern were generally normal in adult induced old $P d g f b^{\text {iECKO }}$ brain vasculature, while hotspot leakage sites showed abnormalities in both CDH5 and CLDN5 expression and distribution. Taken together, adult loss of pericytes did not affect general endothelial arterio-venous hierarchy or EC junctions, although both were disturbed at focal hotspot leakage sites.

We found upregulation of growth factors that are normally expressed during vascular development and in $P d g f b^{\text {ret/ret }}$ mice also in adult-induced $P d g f b^{\text {iECKO }}$ brain vasculature. In further similarity to $P d g f b^{\text {ret } / \text { ret }}$, ANGPT2 was not expressed at hotspot leakage sites in
$P d g f b^{\text {iECKO }}$ brains, but rather in pericyte-deficient vascular stretches without leakage. This suggests that ANGPT2 plays a protective role for the BBB in adult-induced $P d g f b^{\text {iECKO }}$ similar to the situation in $P d g f b^{\text {ret/ret }}$ mice. ${ }^{15}$ ICAM1 was upregulated already in the young brain and retinal capillaries devoid of pericytes, indicating early focal vascular activation. In old brains ICAM1 upregulation also coincided with hotspot leakage sites.

A second unexpected finding was the lack of nodular microvascular calcifications in the brains of adult-induced $P d g f b^{\text {iECKO }}$ mice. Vessel-associated calcifications with a pattern resembling human PFBC have previously been reported in constitutive hypomorphic mutations in $P d g f b .{ }^{9}$ Despite an exhaustive search, we found only a single nodular brain calcification in a single $P d g f b^{\mathrm{iECKO}}$ mouse. Brain vascular calcification is associated with several brain diseases as well as in aging in humans, and our singular finding therefore neither confirms nor refutes a causal link to $P d g f b$. Regardless, given the abundant vascular calcification observed in $P d g f b^{\text {ret/ret }}$ mice, it seems reasonable to assume that its development and progression during adult age requires PDGFB deficiency from young age. In summary, our study discriminates between developmental and homeostatic roles for $P d g f b$ and pericytes in the brain. The latter may be relevant for the several neuro-pathological conditions in which acquired pericyte loss has been reported. ${ }^{31-37}$

\section{Funding}

The author(s) disclosed receipt of the following financial support for the research, authorship, and/or publication of this article: Swedish Research Council (C.B.: 2015-00550), the European Research Council (C.B.: AdG294556), the Leducq Foundation (C.B.: 14CVD02), Swedish Cancer Society (C.B.:150735), Knut and Alice Wallenberg Foundation (C.B.: 2015.0030), Innovative Medicines Initiative (C.B.: IM2PACT-807015).

\section{Acknowledgements}

The authors thank the Rudbeck Animal house personnel for excellent animal care, and C. Olsson, H. Leksell, P. Peterson, and J. Chmielniakova for technical assistance.

\section{Declaration of conflicting interests}

The author(s) declared no potential conflicts of interest with respect to the research, authorship, and/or publication of this article.

\section{Authors' contributions}

M.A.M., A.K., and C.B. conceived and designed the project. E.V.L., K.N., M.A.M., G.B. and A.K. performed experiments. M.A.M., E.V.L. and K.N. wrote the manuscript 
with substantial input from A.K. and C.B. All authors reviewed the manuscript.

\section{Supplemental material}

Supplemental material for this article is available online.

\section{ORCID iDs}

Elisa Vazquez-Liebanas (D) https://orcid.org/0000-0002-79116174

Annika Keller (DD https://orcid.org/0000-0003-1466-3633

Maarja Andaloussi Mäe (D) https://orcid.org/0000-00016985-4379

\section{References}

1. Heldin CH and Westermark B. Mechanism of action and in vivo role of platelet-derived growth factor. Physiol Rev 1999; 79: 1283-1316.

2. Dalla-Favera R, Gallo RC, Giallongo A, et al. Chromosomal localization of the human homolog (c-sis) of the simian sarcoma virus onc gene. Science 1982; 218: 686-688.

3. Johnsson A, Heldin $\mathrm{CH}$, Wasteson A, et al. The c-sis gene encodes a precursor of the $\mathrm{B}$ chain of plateletderived growth factor. Embo J 1984; 3: 921-928.

4. Leveen P, Pekny M, Gebre-Medhin S, et al. Mice deficient for PDGF B show renal, cardiovascular, and hematological abnormalities. Genes Dev 1994; 8: 1875-1887.

5. Soriano P. Abnormal kidney development and hematological disorders in PDGF beta-receptor mutant mice. Genes Dev 1994; 8: 1888-1896.

6. Hellstrom M, Kalen M, Lindahl P, et al. Role of PDGF$\mathrm{B}$ and PDGFR-beta in recruitment of vascular smooth muscle cells and pericytes during embryonic blood vessel formation in the mouse. Development 1999; 126: 3047-3055.

7. Lindahl P, Johansson BR, Leveen P, et al. Pericyte loss and microaneurysm formation in PDGF-B-deficient mice. Science 1997; 277: 242-245.

8. Manyam BV. What is and what is not 'Fahr's disease'. Parkinsonism Relat Disord 2005; 11: 73-80.

9. Keller A, Westenberger A, Sobrido MJ, et al. Mutations in the gene encoding PDGF-B cause brain calcifications in humans and mice. Nat Genet 2013; 45: 1077-1082.

10. Armulik A, Genove G, Mäe M, et al. Pericytes regulate the blood-brain barrier. Nature 2010; 468: 557-561.

11. De La Fuente AG, Lange S, Silva ME, et al. Pericytes stimulate oligodendrocyte progenitor cell differentiation during CNS remyelination. Cell Rep 2017; 20 : 1755-1764.

12. Lindblom $\mathrm{P}$, Gerhardt $\mathrm{H}$, Liebner $\mathrm{S}$, et al. Endothelial PDGF-B retention is required for proper investment of pericytes in the microvessel wall. Genes Dev 2003; 17: 1835-1840.

13. Nahar K, Lebouvier T, Andaloussi Mae M, et al. Astrocyte-microglial association and matrix composition are common events in the natural history of primary familial brain calcification. Brain Pathol 2020; 30: 446-464.
14. Zarb Y, Weber-Stadlbauer U, Kirschenbaum D, et al. Ossified blood vessels in primary familial brain calcification elicit a neurotoxic astrocyte response. Brain 2019; 142: 885-902.

15. Mäe MA, He L, Nordling S, et al. Single-Cell analysis of Blood-Brain barrier response to pericyte loss. Circ Res 2021; 128: e46-e62.

16. Ostman A, Andersson M, Betsholtz C, et al. Identification of a cell retention signal in the B-chain of platelet-derived growth factor and in the long splice version of the A-chain. Cell Regul 1991; 2: 503-512.

17. Abramsson A, Kurup S, Busse M, et al. Defective $\mathrm{N}$-sulfation of heparan sulfate proteoglycans limits PDGF-BB binding and pericyte recruitment in vascular development. Genes Dev 2007; 21: 316-331.

18. Vanlandewijck M, He L, Mae MA, et al. A molecular atlas of cell types and zonation in the brain vasculature. Nature 2018; 554: 475-480.

19. Zeisel A, Hochgerner H, Lonnerberg P, et al. Molecular architecture of the mouse nervous system. Cell 2018; 174 : 999-1014 e22.

20. Enge M, Bjarnegard M, Gerhardt H, et al. Endotheliumspecific platelet-derived growth factor-B ablation mimics diabetic retinopathy. Embo $J$ 2002; 21: 4307-4316.

21. Bjarnegard M, Enge M, Norlin J, et al. Endotheliumspecific ablation of PDGFB leads to pericyte loss and glomerular, cardiac and placental abnormalities. Development 2004; 131: 1847-1857.

22. Park DY, Lee J, Kim J, et al. Plastic roles of pericytes in the blood-retinal barrier. Nat Commun 2017; 8: 15296.

23. Pitulescu ME, Schmidt I, Benedito R, et al. Inducible gene targeting in the neonatal vasculature and analysis of retinal angiogenesis in mice. Nat Protoc 2010; 5: 1518-1534.

24. Percie Du Sert N, Hurst V, Ahluwalia A, et al. The ARRIVE guidelines 2.0: Updated guidelines for reporting animal research. J Cereb Blood Flow Metab 2020; 40: 1769-1777.

25. Vanlandewijck M, Lebouvier T, Andaloussi Mae M, et al. Functional characterization of germline mutations in PDGFB and PDGFRB in primary familial brain calcification. PLoS One 2015; 10: e0143407.

26. Gerhardt $H$, Golding $M$, Fruttiger $M$, et al. VEGF guides angiogenic sprouting utilizing endothelial tip cell filopodia. J Cell Biol 2003; 161: 1163-1177.

27. Henshall TL, Keller A, He L, et al. Notch3 is necessary for blood vessel integrity in the Central nervous system. Arterioscler Thromb Vasc Biol 2015; 35: 409-420.

28. Zarb Y, Sridhar S, Nassiri S, et al. Microglia control small vessel calcification via TREM2. Sci Adv 2021; 7

29. Torok O, Schreiner B, Schaffenrath J, et al. Pericytes regulate vascular immune homeostasis in the CNS. Proc Natl Acad Sci U S A 2021; 118

30. Bell RD, Winkler EA, Sagare AP, et al. Pericytes control key neurovascular functions and neuronal phenotype in the adult brain and during brain aging. Neuron 2010; 68: 409-427.

31. Fernandez-Klett F and Priller J. Diverse functions of pericytes in cerebral blood flow regulation and ischemia. J Cereb Blood Flow Metab 2015; 35: 883-887. 
32. Halliday MR, Rege SV, Ma Q, et al. Accelerated pericyte degeneration and blood-brain barrier breakdown in apolipoprotein E4 carriers with alzheimer's disease. J Cereb Blood Flow Metab 2016; 36: 216-227.

33. Kusuhara S, Fukushima Y, Ogura S, et al. Pathophysiology of diabetic retinopathy: the old and the new. Diabetes Metab J 2018; 42: 364-376.

34. Miners JS, Schulz I and Love S. Differing associations between abeta accumulation, hypoperfusion, blood-brain barrier dysfunction and loss of PDGFRB pericyte marker in the precuneus and parietal white matter in alzheimer's disease. J Cereb Blood Flow Metab 2018; 38: 103-115.
35. Montagne A, Barnes SR, Sweeney MD, et al. Bloodbrain barrier breakdown in the aging human hippocampus. Neuron 2015; 85: 296-302.

36. Sengillo JD, Winkler EA, Walker CT, et al. Deficiency in mural vascular cells coincides with blood-brain barrier disruption in alzheimer's disease. Brain Pathol 2013; 23: 303-310.

37. Winkler EA, Sengillo JD, Sullivan JS, Henkel JS, et al. Blood-spinal cord barrier breakdown and pericyte reductions in amyotrophic lateral sclerosis. Acta Neuropathol 2013; 125: 111-120. 